\title{
Repercusión del acceso vascular sobre la calidad de vida de los pacientes en tratamiento con hemodiálisis
}

Raquel Pelayo Alonso ${ }^{1}$ - José Luis Cobo Sánchez ${ }^{2}$ Marta Reyero López ${ }^{1}$ - Araceli Sáenz de Buruaga Perea ${ }^{1}$ - Alicia Tovar Rincón ${ }^{1}$ - Rosa Alonso Nates ${ }^{1}$ - Ana Begines Ramírez ${ }^{1}$ - Ma Teresa Sola García $^{1}$ - Alexandra Cano Gil ${ }^{1}$

${ }^{1}$ Diplomados en Enfermería

${ }^{2}$ Máster en Ciencias de la Enfermería

Servicio de Nefrología. Hospital Universitario Marqués de Valdecilla. Santander

\section{Resumen}

En todo paciente diagnosticado de insuficiencia renal crónia es imprescindible facilitar un tratamiento adecuado y evitar complicaciones derivadas. Solucionada esta premisa la atención se dirige a ayudar a estos pacientes a vivir lo mejor posible con su patología, en definitiva, mejorar su calidad de vida.

Si el acceso vascular es clave para un buen tratamiento dialítico, podríamos pensar que sería un factor que pudiera influir en la calidad de vida del enfermo renal.

Objetivo: Determinar en qué medida influye el acceso vascular sobre la calidad de vida relacionada con la salud de los pacientes en hemodiálisis.

Metodología: Estudio descriptivo transversal en una población de 38 pacientes en programa de hemodiálisis. Para la evaluación del estado de salud percibido se utilizó la escala visual analógica del Euroqol 5D. Se solicitó a los pacientes que indicasen en la misma

Dirección para la correspondencia:

Raquel Pelayo Alonso

Servicio de Nefrología

Hospital Universitario Marqués de Valdecilla

Avda. Valdecilla s/n 39008 Santander. Cantabria e-mail: sanesteban6@yahoo.es su estado de salud en el momento actual y sin los inconvenientes e incomodidades que les supone su acceso vascular.

Resultados: Aquellos pacientes que se dializaban a través de fístula arteriovenosa interna alcanzaron una puntuación media en la EVA de 58,75 $\pm 20,31$ frente a $60,56 \pm 17,39$ puntos que alcanzaron los portadores de catéteres venosos centrales, no resultando estadísticamente significativo.

Conclusiones: Según nuestro estudio, no existe una influencia significativa del acceso vascular sobre la calidad de vida percibida por el paciente. Los mayores inconvenientes e incomodidades relacionados con el acceso vascular se producen en los portadores de catéteres venosos centrales que llevan más de 1 año en hemodiálisis.

\section{PALABRAS CLAVE:}

- CALIDAD DE VIDA

- FÍSTULA ARTERIOVENOSA

- CATÉTER DE PERMANENCIA

- HEMODIÁLISIS 
Vascular access impact on quality of life of patients treated with hemodialysis

\begin{abstract}
In all patients diagnosed with chronic renal failure is essential to facilitate an adequate treatment and to prevent complications. When this premise is resolved, the attention is directed to assist these patients to live as well as possible with their disease, improving their quality of life ultimately.
\end{abstract}

Objective: To determine the extent vascular access influences the health related quality of life related of hemodialysis patients.

Methodology: Cross-sectional study in a population of 38 hemodialysis patients. Euroqol 5D visual analogue scale was used to evaluate their perceived health status. Patients were asked about their health status at present time, and without the inconvenience and discomfort which gives them their vascular access.

Results: Visual analogue scale mean score of patient with an arteriovenous fistula was $58.75 \pm 20.31$ points vs60.56 \pm 17.39 points of patients with central venous catheter (no statistically significant).

Conclusions: According our study, there is no significant influence of the vascular access on the patient's perceived quality of life. The major inconveniences and discomfort associated with vascular access occur in patients with a central venous catheter, who are treated with hemodialysis more than 1 year.

\section{KEY WORDS:}

- QUALITY OF LIFE

- ARTERIOVENOUS FISTULA

- CATHETERS INDWELLING

- HEMODIALYSIS

\section{Introducción}

La Insuficiencia Renal Crónica (IRC) es una patología cada vez más frecuente, debido especialmente, al continuo envejecimiento de la población. La atención sanitaria de la IRC que precisa tratamiento de sustitución de la función renal, está aumentando considerablemente en torno al 5-10\% cada año según recientes estudios ${ }^{1}$. Los importantes cambios que se han experimentado debido al progreso de las técnicas de depuración extrarrenal desde los años 90 han provocado que los pacientes incluídos en programas de diálisis sean en la actualidad 10-15 años mayores. Esto trae asociado una mayor comorbilidad, con patologías como diabetes, hipertensión arterial, cardiopatía isquémica, vasculopatía periférica... que contribuyen a un peor estado del árbol vascular, principal causa de la imposibilidad de disponer de un acceso vascular para el tratamiento con diálisis².

En todo paciente diagnosticado de IRC es imprescindible facilitar un tratamiento adecuado y evitar complicaciones derivadas. Una vez solucionada esta premisa la atención se dirige a ayudar a estos pacientes a vivir lo mejor posible con su patología, ya que tanto la enfermedad como su tratamiento influyen sobre la afectación de la salud física y mental, la independencia, el bienestar general, las relaciones sociales... En definitiva, se intenta mejorar su calidad de vida ${ }^{3.11}$. La OMS define Calidad de Vida Relacionada con la Salud (CVRS) como la "percepción del individuo de su posición en la vida en el contexto de la cultura y sistema de valores en los que vive y en relación con sus objetivos, expectativas, estándares y preocupaciones" ${ }^{\prime \prime}$. Patrick y Ericsson, en 1996 definieron la CVRS como "el valor asignado por los individuos, grupos de individuos o la sociedad a la duración de la vida modificada por las deficiencias, el estado funcional, la percepción y las opotunidades sociales, que están influidas por una enfermedad, accidente, tratamiento o política" ${ }^{\prime \prime}$.

Los primeros estudios que valoraban la calidad de vida (CV) en el paciente con IRC datan de 1970. Desde entonces, muchos han sido los trabajos realizados pero con resultados poco uniformes, algunos autores encuentran un deterioro significativo en la CV de los pacientes con IRC frente a la población sana, mientras que en otros estudios las diferencias son mínimas ${ }^{4}$. La explicación a esta variación de resultados puede deberse a las diferentes fases por las que pasa el paciente renal 
durante el progreso de su enfermedad, las diferentes modalidades de tratamiento e incluso, la alternancia de estos tratamientos en un corto período de tiempo.

El acceso vascular (AV) es la clave de un tratamiento adecuado ya que representa una de las principales causas de morbilidad, hospitalización y coste en los enfermos tratados con hemodiálisis (HD). Pese al alto porcentaje de pacientes que eligen la HD como alternativa de tratamiento, son muchos, aproximadamente el $50 \%$ los que acceden a ella sin un AV adecuado ${ }^{12,13}$.

En los últimos años se ha visto un aumento en el uso de catéteres venosos centrales (CVC) como AV permanente. El aumento de la edad y de patologías que afectan al lecho vascular como la diabetes, enfermedad vascular periférica, insuficiencia cardíaca, arterioesclerosis, etc; o tratamientos farmacológicos como los inmunosupresores han propiciado esta tendencia. A esto hay que añadir la aparición de complicaciones propias de las fístulas arteriovenosas internas (FAVI) como trombosis, edemas, síndrome de robo, etc. que hacen que el CVC, en algunos casos, sea la única opción de tratamiento ${ }^{14-16}$.

Muchas son las variables que influyen sobre la calidad de vida de los pacientes en HD: edad avanzada, sexo, patologías asociadas, tiempo en diálisis, días de hospitalización, cifras analíticas ${ }^{1,3,7,10,11,17}$. Sin embargo, en pocas ocasiones se hace referencia al acceso vascular del paciente como variable independiente para la CVRS. Si el acceso vascular es clave para un buen tratamiento dialítico, podríamos pensar que sería un factor que pudiera influir en la calidad de vida del enfermo renal.

\section{Objetivo}

Determinar en qué medida influye el acceso vascular sobre la salud percibida o calidad de vida relacionada con la salud (CVRS) de los pacientes en hemodiálisis.

\section{Metodología}

Se realizó un estudio descriptivo transversal. La muestra del estudio incluía un total de 47 pacientes en programa de HD que acuden a nuestro centro 3 veces por semana. Como criterios de exlusión se determinan:
- Llevar menos de 3 meses dializándose a través del mismo AV.

- Presentar situación clínica grave o deterioro cognitivo severo que impida cumplimentar el cuestionario.

- Negativa expresa del paciente.

De la muestra inicial, se excluyeron 3 pacientes por llevar menos de 3 meses dializándose a través del mismo AV, 2 pacientes por presentar situación clínica grave y 4 por deterioro cognitivo, con lo que la muestra final para el estudio fue de 38 pacientes. A todos los pacientes se les solició el consetimiento informado.Ningún paciente se negó a ser incluido en el estudio. Se recogieron datos sociodemográficos (edad y sexo) y variables clínicas, como la causa de IRC (según código EDTA), el tiempo en meses en tratamiento renal sustitutivo (TRS) así como en HD, el índice de comorbilidad y características del AV (si es FAVI o CVC, edad en meses del acceso vascular, existencia de complicaciones así como el uso anterior de otra modalidad de AV).

Para evaluar la comorbilidad se utilizó el índice de comorbilidad de Charlson modificado para pacientes en diálisis. Esta escala fue modificada por Beddhu et a ${ }^{18}$ el año 2000 ya que en la escala original la enfermedad renal se puntúa. Clasifica los pacientes según su comorbilidad en 4 grupos:

1. Baja comorbilidad ( $\leq 3$ puntos).

2. Comorbilidad moderada (4-5 puntos).

3. Comorbilidad alta (6-7 puntos).

4. Gran comorbilidad ( $\geq 8$ puntos).

Para la evaluación del estado de salud percibido se utilizó la escala visual analógica (EVA) del Euroqol 5D (EQ-5D), validada por Badia y cols para población española ${ }^{19}$. Se trata de una EVA vertical de $20 \mathrm{~cm}$ de longitud, graduada de 0 a 100 y con los rótulos "peor estado de salud imaginable" y "mejor estado de salud imaginable" en las puntuaciones 0 y 100, respectivamente. Se solicitó a los pacientes que indicasen en la misma su estado de salud en el momento actual, y posteriormente que indicasen su estado de salud sin los inconvenientes e incomodidades que les supone su acceso vascular. Además, se añadió una pregunta cerrada sobre qué acceso vascular prefería cada paciente. 
Los datos recogidos fueron analizados mediante el paquete estadístico SPSS versión 15.0 para Windows. Se realizó un análisis descriptivo de las variables estudiadas (media, desviación típica y porcentajes). Para la inferencia estadística se utilizaron los test $U$ de MannWhitney, Kruskal-Wallis y Chi-cuadrado. Los resultados se consideraron significativos si el nivel crítico observado era inferior al $5 \%(p<0,05)$. Para el contraste multivariante se utilizó el modelo lineal general.

\section{Resultados}

Se estudiaron 38 pacientes, de los cuales el $66 \%$ eran hombres, con una edad media de $65 \pm 14$ años. La nefropatía más habitual fue la de origen vascular $(28,9 \%)$, seguida de la glomerular $(26,3 \%)$, sistémica $(21,1 \%)$, intersticial $(7,9 \%)$ y otras $(15,8 \%)$. EI $53 \%$ era portador de una FAVI y el $47 \%$ de un CVC. Sólo 6 pacientes $(15,8 \%)$ presentaron algún tipo de complicación relacionada con su AV: 2 mal flujo y 4 punción dificultosa de la FAVI. Entre los pacientes con FAVI el $60 \%$ había tenido una FAVI previa y el $40 \%$ había tenido un catéter previo; entre los pacientes con CVC el 39\% habían tenido una FAVI previa y el 61\% habían tenido un catéter previo. La media de tiempo en tratamiento sustitutivo renal fue de $91 \pm 109$ meses, y en concreto en HD de $44 \pm 47$ meses. Los pacientes con FAVI llevaban más tiempo en HD que los pacientes con CVC (59 vs 28 meses de media). El tiempo medio de duración de las FAVIs fue de 78 meses, y de 13 meses en el caso de los CVCs. La puntuación media del índice de comorbilidad de Charlson fue de 5,5 $\pm 1,9$ puntos: 4,9 puntos para los portadores de FAVI y 6,17 puntos para los CVCs $(p=0,038)$. El número de pacientes según grado de comorbilidad y tipo de acceso vascular puede observarse en el gráfico 1.

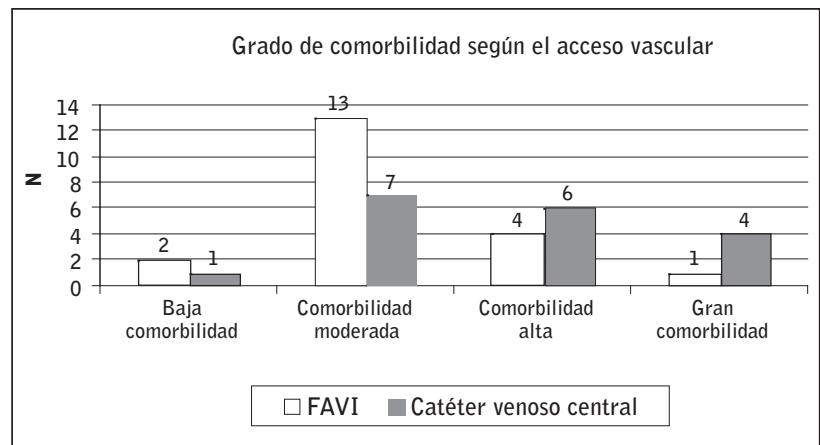

Gráfico 1. Número de pacientes según grado de comorbilidad y acceso vascular
Todos los pacientes portadores de FAVI no preferirían otro acceso vascular, mientras que el $28 \%$ de los portadores de un CVC preferirían tener una FAVI.

La puntuación media de EVA en el momento de la valoración, para el total de los pacientes, alcanzó los $59,61 \pm 18,78$ puntos, siendo $58,75 \pm 20,31$ la puntuación alcanzada por los portadores de FAVI frente a los $60,56 \pm 17,39$ puntos que reflejaron aquellos que se dializaban a través de CVC (Gráfico 2).

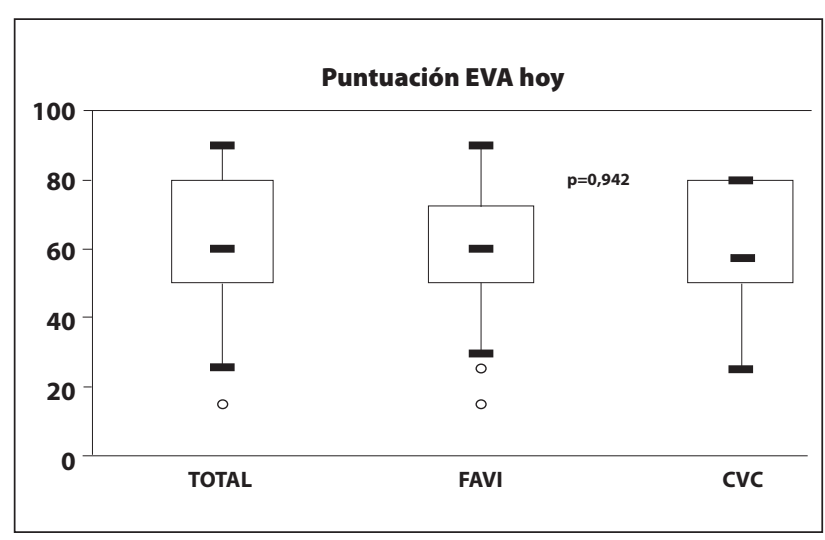

Gráfico 2. Puntuación de la escala visual analógica (EVA) en el momento de la valoración, para la totalidad de la muestra y según el acceso vascular (FAVI: fístula arteriovenosa; CVC: catéter venoso central)

Todos los pacientes puntuaron mejor su estado de salud sin los inconvenientes del AV siendo 68,8 21,72 la puntuación alcanzada por los pacientes con FAVI y $69,94 \pm 12,8$ la obtenida por los portadores de CVC (Gráfico 3).

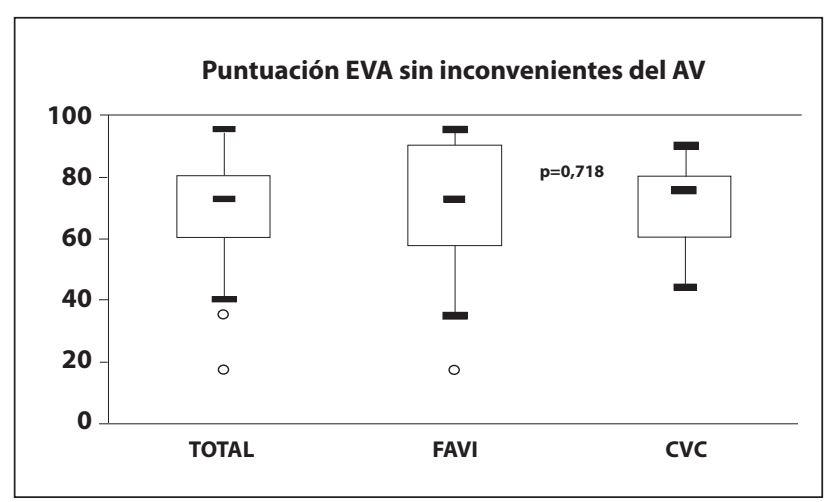

Gráfico 3. Puntuación de la escala virtual analógica (EVA) sin los inconvenientes atribuibles al acceso vascular, para la totalidad de la muestra y según el acceso vascular (FAVI: fístula arteriovenosa; CVC: catéter venoso central) 
El gráfico 4 hace referencia a la diferencia entre las puntuaciones EVA, es decir, aquella que se atribuye a los inconvenientes de cada acceso vascular, siendo de $11,05 \pm 11,79$ para las FAVIs y $12,17 \pm 10,07$ para los CVCs.

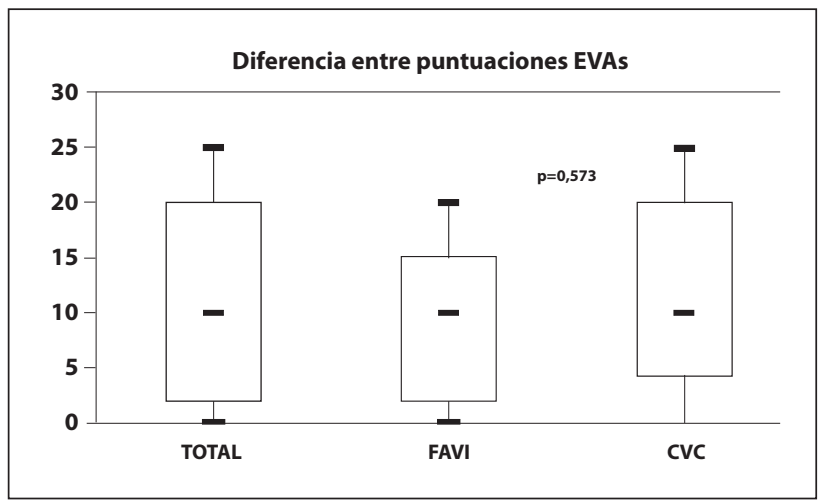

Gráfico 4. Diferencia entre las dos puntuaciones de la escala virtual analógica (EVA) para la totalidad de la muestra y según el accweso vascular (FAVI: fístula ateriovenosa; CVC: catéter venoso central)

No hubo diferencias estadísticamente significativas entre las puntuaciones de la EVA para su salud actual, ni para la puntuación de la EVA sin los inconvenientes del $A V$, ni para la diferencia entre ambas. Tampoco hubo diferencias estadísticamente significativas en la percepción de su estado de salud, para ambos casos, según el resto de variables (edad, sexo, complicaciones del acceso vascular, querer otro acceso vascular, nefropatía, grado de comorbilidad).

En la tabla 1 se determinan las diferentes puntuaciones de EVA según el AV y el grupo de edad (mayor o menor de 65 años). De los pacientes con FAVI, la mitad eran mayores 65 años $(n=10)$ y la otra mitad menores de 65 años $(n=10)$. Entre los pacientes con catéter el $22 \%(n=4)$ eran menores de 65 años y el $78 \%(n=14)$ mayores de 65 años.

Los datos de puntuación EVA según el AV y el tiempo en HD se pueden ver en la tabla 2. Cabe decir que todos los pacientes portadores de FAVI Ilevaban más de 1 año en programa de HD. Entre los pacientes con catéter, 11 llevaban más de un año en HD y 7 menos de uno.

\section{Discusión}

Durante los últimos años, los aspectos psicosociales del enfermo renal han empezado a adquirir mayor importancia dentro de la valoración del paciente renal, siendo la CVRS uno de los más valorados; pero los diferentes conceptos de CVRS, las múltiples variables

\begin{tabular}{|l|c|c|c|c|c|}
\hline & \multicolumn{3}{|c|}{ FAVI } & \multicolumn{2}{c|}{ CVC } \\
\hline & $<65$ años & $>65$ años & $<65$ años & $>65$ años \\
\hline Puntuación EVA hoy & $60,5 \pm 7,3$ & $57 \pm 5,63$ & $60 \pm 9,12$ & $60,71 \pm 4,77$ & $<0,001$ \\
\hline $\begin{array}{l}\text { Puntuación EVA sin los } \\
\text { inconvenientes del AV }\end{array}$ & $75,2 \pm 7,42$ & $62,4 \pm 5,94$ & $69,75 \pm 9,57$ & $70 \pm 3,056$ & $<0,01$ \\
\hline Diferencia EVAs & $14,7 \pm 4,67$ & $7,4 \pm 2,13$ & $14,75 \pm 4,75$ & $11,43 \pm 2,79$ & 0,024 \\
\hline
\end{tabular}

Tabla 1: Puntuación de la escala visual analógica (EVA) según el acceso vascular y el grupo de edad (AV: acceso vascular; FAVI: fístula arteriovenosa; CVC: catéter venoso central)

\begin{tabular}{|l|c|c|c|c|c|}
\hline & \multicolumn{2}{|c|}{ FAVI } & \multicolumn{2}{c|}{ CVC } \\
\hline & $<\mathbf{l}$ año HD & $>1$ año HD & $<1$ año HD & $>1$ año HD \\
\hline Puntuación EVA hoy & - & $58,75 \pm 4,54$ & $67,14 \pm 6,44$ & $56,36 \pm 5,13$ & 0,001 \\
\hline $\begin{array}{l}\text { Puntuación EVA sin los } \\
\text { inconvenientes del AV }\end{array}$ & - & $68,8 \pm 4,85$ & $67,71 \pm 5,54$ & $71,3 \pm 3,63$ & 0,015 \\
\hline Diferencia EVAs & - & $11,05 \pm 2,63$ & $4,86 \pm 2,67$ & $16,82 \pm 2,72$ & 0,163 \\
\hline
\end{tabular}

Tabla 2: Puntuación de la escala visual analógica (EVA) según el acceso vascular y el tiempo en tratamiento con hemodiálisis (AV: acceso vascular; FAVI: fístula arteriovenosa; CVC: catéter venoso central; HD: hemodiálisis) 
que se valoran como posibles factores influyentes así como los diferentes instrumentos de medida justifican la variabilidad de los resultados 3,7,10,11,17.

Cuestionarios como el SF-36, o el KDQOL han sido ampliamente utilizados. Sin embargo el EQ-5D del que se ha extraído la escala visual analógica (EVA) utilizada en el presente estudio, es en general poco usado pese a ofrecer ciertas ventajas: es rápido, sencillo y la EVA ofrece poca carga de trabajo para el paciente por su fácil comprensión lo que repercute de forma positiva en la calidad y cantidad de datos recogidos, disminuyendo las respuestas perdidas o equivocadas ${ }^{10,15}$.

En nuestro estudio se obtiene una puntuación media en la EVA de $59,61 \pm 18,78$ puntos, similar al resultado obtenido en otro estudio realizado en 455 pacientes sometidos a hemodiálisis, dónde la puntuación obtenida alcanzaba los $60,14 \pm 18$ puntos $^{10}$. Analizando los datos según el AV, los pacientes portadores de FAVI reflejan una puntuación en la EVA basal menor que los portadores de CVC. Wasse y cols. ${ }^{15}$ estudiaron mediante la misma escala un total de 647 pacientes sometidos a HD y observaron que los pacientes portadores de FAVI reflejaban mayor puntuación en la EVA que los portadores de CVC. Sin embargo, en el estudio de Seguí y cols. ${ }^{17}$ no se observan diferencias significativas al comparar ambos AVs, aunque en este caso, la valoración de la CV se realizó con el cuestionario SF-36. A pesar de obtener mayor puntuación en la EVA, también destaca que son los pacientes portadores de CVC los que reflejan un mayor peso de los inconvenientes de su acceso vascular (AV) sobre su calidad de vida $(12,7 \pm 10,07$ vs $11,05 \pm 11,79$ respectivamente). Cabe recordar, que nuestro estudio no valora datos analíticos ni físicos, solamente la percepción subjetiva por parte del paciente sobre su CV.

A pesar de obtener mayor puntuación en la EVA, también destaca que son los pacientes portadores de CVC los que reflejan un mayor peso de los inconvenientes de su AV sobre su calidad de vida.

Según algunas publicaciones, se produce una disminución de la capacidad funcional del paciente sometido a HD, sin embargo, la capacidad psicológica de adaptación a la situación se mantiene, lo que puede explicar que los pacientes de mayor edad reflejen una CVRS ${ }^{10,11}$. Analizando nuestros datos, no se obtienen grandes diferencias en la puntuación EVA entre los pacientes mayores de 65 años y los menores. Sin embargo, si distinguimos según el $\mathrm{AV}$, los que reflejan mayor $\mathrm{CV}$ son los mayores de 65 años portadores de CVC, quizás debido a esa mayor capacidad de adaptación que reflejan otros estudios y a un menor sentimiento de pérdida que los pacientes más jóvenes ${ }^{4}$. Por otro lado, aquellos que reflejan una mayor influencia de los inconvenientes e incomodidades del AV sobre su CV son los menores de 65 años, sin diferencias entre el tipo de AV. Esto puede estar relacionado con la importancia de la apariencia física y la carga estética que el acceso vascular puede generar sobre los pacientes más jóvenes, como ya demostraron otros estudios previos ${ }^{5}$.

Por esta mayor capacidad de adaptación comentada anteriormente, aquellos que llevan mayor tiempo en programa de HD refieren una mayor puntuación de $\mathrm{CVRS}^{4}$. Al analizar nuestros datos, las diferencias entre los que llevan más de 1 año dializándose y los que llevan menos, destaca que son los portadores de CVC con menos de 1 año en programa los que reflejan mejor puntuación, posiblemente por la menor presencia de complicaciones del AV. Esta misma razón puede explicar también, el hecho de que los que refieren mayor influencia de los inconvenientes del AV sobre la CV sean los portadores de CVC que llevan más de 1 año en HD. De estos, podemos deducir la importancia que tiene el cuidar y mantener en las mejores condiciones posibles los accesos vasculares de nuestros pacientes, papel fundamental de la enfermería, ya que como hemos visto, influye en cierta medida sobre su CV, sin olvidar la gran importancia que el AV tiene sobre la morbilidad y mortalidad del enfermo renal ${ }^{4,13,16}$. Sería interesante evaluar estos mismos datos con un mayor margen de tiempo y periodicidad, así como las posibles complicaciones, para valorar los cambios que se producen.

Cabe destacar la distribución de los diferentes AV en los pacientes del estudio, ya que solamente el $53 \%$ son portadores de FAVI frente al $80 \%$ que recomiendan las guías de la Sociedad Española de Nefrología ${ }^{13}$. En los últimos años se han producido cambios en la distribución del AV de los pacientes ${ }^{16}$ aumentando el número de pacientes portadores de CVC frente a los portadores de FAVI, tanto en los pacientes que inician programa de HD como en los prevalentes. Este aumento de CVC está también asociado con un mayor incremento de la población anciana y una mayor comorbilidad. Los resultados de nuestro estudio corroboran esta distri- 
bución del AV, ya que la puntuación media del índice de Charlson para pacientes portadores de CVC es de 6,17 (comorbilidad alta) frente a 4,9 (comorbilidad moderada) para los pacientes con FAVIII . En cuanto a la distribución según la edad, del total de los pacientes con CVC el 78\% son mayores de 65 años. Se puede decir que la edad y la comorbilidad van relacionadas, haciendo que en algunos casos, el CVC sea la única alternativa de acceso vascular ${ }^{13}$.

\section{Conclusiones}

Según nuestro estudio, no existe una influencia significativa del AV sobre la calidad de vida percibida por el paciente.

El acceso vascular de preferencia para la mayoría de los pacientes sometidos a HD es la FAVI.

Los mayores inconvenientes e incomodidades relacionados con el AV se producen en los portadores de CVC que llevan más de 1 año en HD.

Sería interesante realizar más estudios sobre la posible influencia del AV sobre la CV de los pacientes, debido al papel fundamental que la Enfermería tiene sobre el uso, mantenimiento y cuidado del $\mathrm{AV}$, pilar fundamental del tratamiento del paciente sometido a HD.

Recibido: Septiembre 2011

Revisado: Septiembre 2011

Modificado: Octubre 2011

Aceptado: Noviembre 2011

\section{Bibliografía}

1. De Francisco ALM, San Juan F, Foraster A, Fabado S, Carretero D, Santamaría C. y cols. Estudio epidemiológico de pacientes ancianos con insuficiencia renal crónica en hemodiálisis. Nefrología 2008; 28(1): 48-55.

2. Lerma R, Callejas JM. Accesos vasculares para hemodiálisis: equipos multidisciplinarios. Angiología 2005; 57(supl 2): S169-S176.
3. Álvarez-Ude F, Vicente $E$, Badía X. La medida del a calidad de vida relacionada con la salud en los pacientes en programa de hemodiálisis y diálisis peritoneal continua ambulatoria de Segovia. Nefrología 1995; 15(6): 572-580.

4. Martín A, Reig A, Sarró F, Ferrer R, Arenas D, González $F$, y cols. Evaluación de la calidad de vida en pacientes de una unidad de hemodiálisis con el cuestionario Kidney Disease Quality of Life-Short Form (KDQ0L-SF). Dial Trasplant 2004; 25(2): 79-92.

5. Cleary J, Drennan J. Quality of life of patients on haemodialysis for end-stage renal disease. J Adv Nurs 2005; 51(6):577-586.

6. Cidoncha MA, Estévez I, Santiago J, Andueza M, Zubiaga G. Calidad de vida en pacientes en hemodiálisis. Libro de comunicaciones XXVIII Congreso Nacional de la Sociedad Española de Enfermería Nefrológica. Palma de Mallorca. 2003: 14-17. En: SEDEN. Comunicaciones presentadas a los Congresos de la Sociedad Española de Enfermería Nefrológica 1981-2010 [CD-ROM]. Madrid: SEDEN; 2010.

7. Ruiz MJ, Román M, Martín G, Alférez MJ. Calidad de vida relacionada con la salud en las diferentes terapias sustitutivas de la Insuficiencia Renal Crónica. Rev Soc Esp Enferm Nefrol 2003; 6(4): 6-16.

8. WHOQOL Group. Study protocol for the World Health Organization Project to develop a quality of life assessment instrument. Qual Life Res. 1993; 2(2):153-9.

9. Patrick DL, Erickson P. Applications of Health Status Assessment to Health Policy. In: Spilker B, ed. Quality of Life and Pharmacoeconomics in Clinical Trials. Second ed. Philadelphia: Lippincott-Raven Publishers;1996. pp. 717-727.

10. Wasserfallen J, Halabi G, Saudan P, Perneger T, Harold I, Martin P, Wauters J. Quality of life on chronic dialysis: comparison between haemodialysis and peritoneal dialysis. Nephrol Dial Transplant 2004; 19(6): 1594-1599.

11. Rebollo P, Bobes J, Gonzalez MP, Saiz P, Ortega F. Factores asociados a la calidad de vida relacionada 
con la salud de los pacientes en terapia renal sustitutiva. Nefrología 2000; 20(2): 171-181.

12. Rodriguez JA, González E. Accesos vasculares para hemodiálisis: preparación del paciente con insuficiencia renal crónica. Angiología 2005; 57 (supl 2): S11-S21.

13. Remón D; Bardón E; Vila ML. Accesos para comenzar la depuración extrarrenal: vascular y peritoneal. Acceso temporal en prediálisis. Nefrología 2008; 28(supl 3):105-112.

14. Llagostera S, Martínez E. Perspectivas futuras en accesos vasculares. Angiología 2005; 57 (supl 2): S199-S203.

15. Wasse H, Kutner N, Zhag R, Huang Y. Association of initial hemodialysis vascular acess with patient reported health status and quality of life. Clinic $J$ Am Soc Nephrol. 2007; 2(4):708-14.
16. Malek T, Álvarez-Ude F, Gil MT, Moledus A, LópezCollado M. Cambios en el acceso vascular en una unidad de diálisis en los últimos años: ¿problemas de planificación, cambio de preferencias o cambio demográfico? Nefrología 2008; 28(5):531-538.

17. Seguí $A$, Amador $P$, Ramos AB. Calidad de vida en pacientes con insuficiencia renal crónica en tratamiento con diálisis. Rev Soc Esp Enferm Nefrol 2010; 13(3):155-160.

18. Beddhu S, Bruns FJ, Saul M, Seddon P, Zeidel ML. A simple comorbidity scale predicts clinical outcomes and costs in dialysis patients. Am J Med. 2000; 108(8):609-613.

19. Badia X, Schiaffino A, Alonso J, Herdman M. Using the EuroQoI 5-D in the Catalan general population: feasibility and construct validity. Qual Life Res.1998; 7(4): 311- 22. 